\title{
The value of CESM in the evaluation of intraductal breast papilloma: a comparative study with DCE-MRI
}

\author{
Rania Hegazy ${ }^{1 *}$ D, Lamia Adel ${ }^{1}$ and Rabab Yasin ${ }^{2}$
}

\begin{abstract}
Background: The purpose of this study was to assess the accuracy of contrast-enhanced spectral mammography (CESM) as compared to dynamic contrast-enhanced MRI (DCE-MRI) in the evaluation of intraductal breast papilloma with histopathological correlation. This study is a retrospective study done on 45 consecutive patients. Their ages ranged between 36 and 70 years with a mean age of $51.1 \pm 8.7$. All of them were suspected to have intraductal papilloma on full-field digital mammography (FFDM), 3D automated breast ultrasound (3D-ABUS), and 2D handheld ultrasound (2DHHUS). CESM and DCE-MRI were done for all patients. The data were collected, tabulated, statistically analyzed using an IBM personal computer with Statistical Package of Social Science (SPSS).

Results: There were 37 histopathology proven papillomas either by US-guided core needle biopsy, surgical biopsy following wire localization or fine-needle aspiration cytology (FNAC). Lesions are divided into three subgroups according to size. When the size of lesion $>10 \mathrm{~mm} \mathrm{MRI}$ sensitivity and specificity is $100 \%$ and $66.7 \%$, that of CESM is $90.1 \%$ and $0 \%$ respectively. In lesions sized $5-10 \mathrm{~mm}$ Sensitivity and specificity of MRI is $100 \%$ and $58 \%$ respectively and those of CESM are $63.6 \%$ and $50 \%$ respectively. Lesions less than $5 \mathrm{~mm}$ MRI sensitivity and specificity is $100 \%$ and $0 \%$, that of CESM was $0 \%$ and $100 \%$ respectively.
\end{abstract}

Conclusion: DCE-MRI has a significantly higher sensitivity than CESM for the diagnosis of intraductal papilloma whatever the size of the lesion, especially when the size is less than $5 \mathrm{~mm}$.

Keywords: Papilloma, CESM: contrast-enhanced spectral mammography, DCE-MRI: dynamic contrast-enhanced MRI

\section{Background}

Intraductal papilloma is a benign tumor which occurs as a result of epithelial and myoepithelial cell proliferation over fibrovascular stalk forming an arborescent structure inside the duct lumen [1]. Regarding its location, it is either a central papilloma which arises in the retroareolar region in a large duct or a peripheral papilloma which arises in terminal ducts. Central papilloma is usually symptomatic with nipple discharge while the peripheral papilloma is mostly asymptomatic [1].

There are different varieties of intraductal papillomas, all of them grossly having a papillary appearance and are considered as solitary, multiple or juvenile papillomatosis [2].

\footnotetext{
* Correspondence: raniahegazy@hotmail.com; hegazyrania@gmail.com ${ }^{1}$ Radiology Department, Faculty of Medicine, Cairo University, Cairo, Egypt Full list of author information is available at the end of the article
}

Solitary papilloma is more common and mostly seen in a central location. Multiple papillomas arise from the terminal ductal lobular unit, so they usually present as palpable mass and not nipple discharge. Associated atypical ductal hyperplasia (ADH), atypical lobular hyperplasia (ALH), ductal carcinoma in-situ (DCIS), or malignancy occurs in about $80.4 \%$ of multiple papillomas [3].

Intraductal papilloma is usually seen in premenopausal women, age range 30-50 years old. It usually presents with nipple discharge (bloody, serous, or sero-sanginous) that causes anxiety and discomfort in many cases [3].

Nipple discharge is considered the third most common complaint after breast pain and breast mass with a prevalence of $4.8-7.4 \%$. It is clinically significant when it is persistent, spontaneous, and in non-lactating women [2].

Different breast imaging modalities are sometimes useful in detecting an intraductal papilloma, however, uncertainties exist with each modality. The imaging 
modalities for intraductal lesion include mammography, ultrasonography, magnetic resonance imaging, and ductography $[2,4,5]$.

Researches on DCE-MRI have shown that although wash-in and wash-out curves are able to differentiate benign from malignant breast lesions, it has no significant role in differentiating intraductal papillomas from invasive carcinomas $[1,6,7]$.

Contrast-enhanced spectral mammography (CESM) has recently been used to increase the sensitivity of mammography for detecting breast lesions based on its ability to image neovascularity similar to MRI [8-11].

To our knowledge, very few studies have been conducted to evaluate the role of CESM in the assessment of intraductal lesions. In this study, we aim to characterize imaging findings of intraductal papillomas and other intraductal lesions in CESM as compared to DCE-MRI and to assess if CESM can differentiate benign from malignant intraductal lesions.

\section{Methods}

This study is a retrospective study done on 45 patients referred to the radiology department from February 2018 to July 2019, who were suspected to have intraductal papilloma on full-field digital mammography (FFDM) and ultrasound (US) examinations. Their ages ranged between 36 and 70 years with a mean age of $51.1 \pm 8.7$. CESM and DCE-MRI were done for all the patients.

\section{CESM technique}

All our patients $(n=45)$ underwent bilateral CESM using GE Senographe ${ }^{\text {ma }}$ Essential with SenoBright upgrade.

\section{Technique}

CESM was done in the presence of two radiographers with 6-14 years of experience in mammography to assure speed and consistency with a radiologist in attendance to monitor patient and images. Patients were asked to present their renal function profile on the examination day, after having ensured that there was no history of allergy or contraindications to I.V. contrast administration. The procedure was explained to the patients and written consent obtained and an IV line secured. With the patient wearing the examination gown and seated comfortably in the mammography room, $1.5 \mathrm{cc} / \mathrm{kg}$

Table 1 represented the clinical presentation of our patients

\begin{tabular}{lll}
\hline Clinical presentation & Number of patients & $\%$ \\
\hline Nipple discharge & 28 & $65 \%$ \\
Palpable mass & 10 & $23 \%$ \\
Nipple discharge with palpable mass & 7 & $16 \%$ \\
Asymptomatic & 7 & $16 \%$ \\
Total & 45 & $100 \%$ \\
\hline
\end{tabular}

Table 2 represented the histopathological results of our patients

\begin{tabular}{lll}
\hline Histpathological results & $\mathrm{N}$ & $\%$ \\
\hline Intraductal papilloma & 37 & $82.4 \%$ \\
IDC & 1 & $2.2 \%$ \\
Intraductal papillary cystic carcinoma & 1 & $2.2 \%$ \\
Fibroadenoma & 1 & $2.2 \%$ \\
Granulomatous mastitis & 1 & $2.2 \%$ \\
Organized hematoma with blood clot & 2 & $4.4 \%$ \\
False result with foci of parenchymal enhancement & 2 & $4.4 \%$ \\
Total & 45 cases & $100 \%$ \\
\hline
\end{tabular}

320mg\% Visapaque (Iodixamol) which had been warmed up to body temperature was given at a flow rate of $3 \mathrm{mls} /$ sec using Imaexon automated injector. Low and high energy digital images were taken of both breasts from minute 2 to minute 6 in CC and MLO projections, starting with the unaffected breast using a built-in stopwatch. CESM image acquisition was in the sequence of CC normal side, CC lesion side, MLO lesion side, and MLO normal side. This was to improve the likelihood of contrast uptake by the lesion. No major complication or contrast reaction occurred in the examined patients. The cannula was then removed, and post-injection instructions were given to the patient.

\section{Image analysis}

Recombined CESM images were then analyzed by 3 radiologists with 34,23 , and 11 years of experience respectively in mammography in terms of lesion enhancement as follows:

No enhancement (negative study). Mild enhancement. Moderate enhancement. Intense/avid enhancement. Each radiologist was blinded to the MRI results and to reports by the other two colleagues. When there was a discrepancy between two radiologists in interpretation, the third radiologist was consulted for reference. A final score would be provided after negotiation. Inter-observer variability was thus reduced to the minimum. Interpretation of breast lesions was assessed regarding (1) presence or absence of a lesion, (2) degree of enhancement, (3) mass or non-mass enhancement (4) shape: regular/irregular, and (5) papilloma/other pathology.

Table 3 Represented analysis of papilloma cases regarding enhancement on CESM

\begin{tabular}{lll}
\hline Degree of enhancement on CESM & $N$ & $\%$ \\
\hline No enhancement & 17 cases & $46 \%$ \\
Faint & 11 cases & $30 \%$ \\
Moderate & 7 cases & $19 \%$ \\
Intense & 2 cases & $5 \%$ \\
Total & 37 & $100 \%$ \\
\hline
\end{tabular}


Table 4 Correlation between mass/non-mass enhancement of papilloma cases on CESM

\begin{tabular}{lllll}
\hline CESM & Mass enhancement & Non-mass enhancement & No enhancement & Total \\
\hline $\begin{array}{l}2(5 \%) \\
\text { cases benign papilloma (size } 15 \mathrm{~mm})\end{array}$ & $\begin{array}{l}18(49 \%) \\
\text { benign papillomas }\end{array}$ & $\begin{array}{l}17(46 \%) \\
\text { papillomas }\end{array}$ & 37 pathologically proven papillomas \\
\end{tabular}

\section{MRI technique}

DCE-MRI breasts were performed on a 1.5-T system (Magnetom Aera; Siemens, Erlangen, Germany). Patients were imaged in a prone position using a dedicated breast coil imaging both sides simultaneously. It is a fourchannel phased-array breast coil. In premenopausal women, the study was done between day 5 and day 12 of the menstrual cycle to minimize the effect of the hormonal change on contrast uptake. All patients signed written consent prior to the study.

An intravenous cannula was fixed before positioning the patient on the MR table. The contrast agent was injected using an automatic MR-compatible power injector. Contrast given was gadobutrol (Gadovist; Bayer-Schering, Berlin, Germany) in a dose of $0.1 \mathrm{mmol} / \mathrm{kg}$ (or $0.2 \mathrm{ml} / \mathrm{kg}$ ) and a flow rate of $2 \mathrm{ml} / \mathrm{s}$ flushed by $20 \mathrm{ml}$ of normal saline and one pre- and six sequential contrast-enhanced images were then acquired, sequence per minute.

MRI sequences were taken with parameters; slice thickness $4 \mathrm{~mm}$, FOV $350 \mathrm{~mm}$ and matrix $512 \times 512$ :

- Axial Turbo Inversion Recovery Magnitude (TIRM) with TR 7700ms, TE 74 ms.

- Axial T2 fast spin-echo (FSE) with TR 6160 ms, TE $76 \mathrm{~ms}$.

- Axial T1 fast spin-echo (FSE) with TR 415 ms, TE $4.6 \mathrm{~ms}$.

- Diffusion-weighted imaging (DWI) was done TR $8200 \mathrm{~ms}$, TR $85 \mathrm{~ms}$ in the axial plane bilaterally with $b$ values of 0,500 , and $1000 \mathrm{~s} / \mathrm{mm}^{2}$.

- ADC map was systematically performed.

- Serial dynamic images acquired six times in the axial plane with three-dimensional transverse fast, low angle shot T1-weighted sequence (TR/TE, 5.27/2.39 ms, FOV $260 \mathrm{~mm}$, matrix, $512 \times 512$; section thickness $1.6 \mathrm{~mm}$ ).

- Then post-processing subtraction of the precontrast images from the post-contrast images with fat suppression, multi-planar reconstruction (MPR), and maximum intensity projections (MIP).

- Bilateral sagittal T1 FSE post contrast administration.

- Delayed axial T1 post-contrast imaging 10 min after contrast injection.

- Three radiologists of 5-18 years of experience in MRI breast analyzed the images.

\section{Results}

This retrospective study was of 45 cases (4 cases with bilateral lesions) referred to radiology. Their ages ranged between 36 and 70 years with a mean age of $51.1 \pm 8.7$. Their presenting symptoms were nipple discharge (bloody/ serous) (28 patients; 65\%), breast lump (10 patients; 23\%), within these two groups those presenting with both discharge and a palpable mass (7 patients; 16\%). Asymptomatic patients came for routine screening (7;16\%) (Table 1).

There were 37 proven papillomas either by US-guided core needle biopsy, excision biopsy, or FNA cytology. The histopathological results of the cases are summarized in Table 2.

The enhancement pattern of the papillomas on CESM is shown in Tables 3 and 4. The contrast uptake of the lesions after IV contrast shows a highly statistical significance between CESM and DCE-MRI (Table 5 ) with $P$ value $\leq 0.001$.

The mean size of lesions was $11.1 \pm 4.6 \mathrm{~mm}$ on DCEMRI and $13.8 \pm 3.4 \mathrm{~mm}$ on CESM with statistical significance $P=0.005^{*}(\leq 0.05)$ (Table 5$)$.

There was no statistical significance between CESM and DCE-MRI (Table 5) as regards the margin of the lesion $(P=0.99)$. The margin of the lesions was irregular in 4 cases on CESM; 2 cases of atypical papilloma, one case of invasive ductal carcinoma (IDC) and one case of granulomatous mastitis.

On DCE-MRI, the irregular margin was seen in 7 cases; 1 case each of intraductal papillary cystic carcinoma, IDC, granulomatous mastitis, and 4 cases of atypical papillomas.

DCE-MRI has high sensitivity for the detection of intraductal papilloma (100\%), but its specificity was low $37.5 \%$. CESM has a lower sensitivity (64.9\%) for the detection of intraductal lesion as compared to DCE-MRI and a higher specificity (50\%) (Table 6). The area under the ROC curve for DCE-MRI $=0.69$ and the area under the curve for CESM $=0.57$.

The lesions were subgrouped into three groups, the first group when the size is larger than $10 \mathrm{~mm}$, the

Table 5 Represents the characteristic of the lesions on CESM \& MRI

\begin{tabular}{|c|c|c|c|c|c|}
\hline Characteristic of mass & \multicolumn{2}{|l|}{$\mathrm{MRI}$} & \multicolumn{2}{|c|}{ CESM } & $P$ value \\
\hline Range of size of lesion & \multicolumn{2}{|c|}{$4-20$} & \multicolumn{2}{|c|}{$7.5-21$} & $\mathrm{~T}=2.9$ \\
\hline Mean size of lesions \pm SD & \multicolumn{2}{|c|}{$11.1 \pm 4.6$} & \multicolumn{2}{|c|}{$13.8 \pm 3.4$} & $P=0.005^{*}(\leq 0.05)$ \\
\hline Contrast uptake & $\mathrm{N}$ & $\%$ & $\mathrm{~N}$ & $\%$ & \\
\hline - Positive & 45 & 100 & 28 & 62.2 & $X^{2}=20.9$ \\
\hline - Negative & 0 & 0 & 17 & 37.8 & $P=0.00^{* *}(\leq 0.001)$ \\
\hline \multicolumn{6}{|l|}{ Shape } \\
\hline - Regular & 39 & 85.7 & 38 & 84.4 & Fisher's Exact $=0.002$ \\
\hline - Irregular & 6 & 14.3 & 7 & 15.6 & $P=0.99(>0.05)$ \\
\hline
\end{tabular}


Table 6 Diagnosis of papilloma by MRI \& CESM

\begin{tabular}{|c|c|c|c|c|c|c|}
\hline & $\begin{array}{l}\text { Positive } \\
\text { by MRI }\end{array}$ & $\begin{array}{l}\text { Negative } \\
\text { by MRI }\end{array}$ & Total & $\begin{array}{l}\text { Positive by } \\
\text { CESM }\end{array}$ & $\begin{array}{l}\text { Negative } \\
\text { by CESM }\end{array}$ & Total \\
\hline Papilloma & 37 & 0 & 37 & 24 & 13 & 37 \\
\hline Non papilloma & 5 & 3 & 8 & 4 & 4 & 8 \\
\hline Total & 37 & 8 & 45 & 28 & 17 & 45 \\
\hline Sensitivity & \multicolumn{3}{|c|}{$(37 / 37) * 100=100 \%$} & \multicolumn{3}{|c|}{$(24 / 37) * 100=64.9 \%$} \\
\hline Specificity & \multicolumn{3}{|c|}{$(3 / 8) * 100=37.5 \%$} & \multicolumn{3}{|c|}{$(4 / 8) * 100=50 \%$} \\
\hline False positive & \multicolumn{3}{|c|}{$(5 / 8) * 100=62.5 \%$} & \multicolumn{3}{|c|}{$(4 / 8) * 100=50 \%$} \\
\hline False negative & \multicolumn{3}{|c|}{$(0 / 37) * 100=0 \%$} & \multicolumn{3}{|c|}{$(13 / 37) * 100=35.1 \%$} \\
\hline $\begin{array}{l}\text { Positive predictive } \\
\text { value }\end{array}$ & \multicolumn{3}{|c|}{$(37 / 42) * 100=88.1 \%$} & \multicolumn{3}{|c|}{$(24 / 28) * 100=85.7 \%$} \\
\hline $\begin{array}{l}\text { Negative predictive } \\
\text { value }\end{array}$ & \multicolumn{3}{|c|}{$(3 / 3)^{*} 100=100 \%$} & \multicolumn{3}{|c|}{$(4 / 17)^{*} 100=23.5 \%$} \\
\hline
\end{tabular}

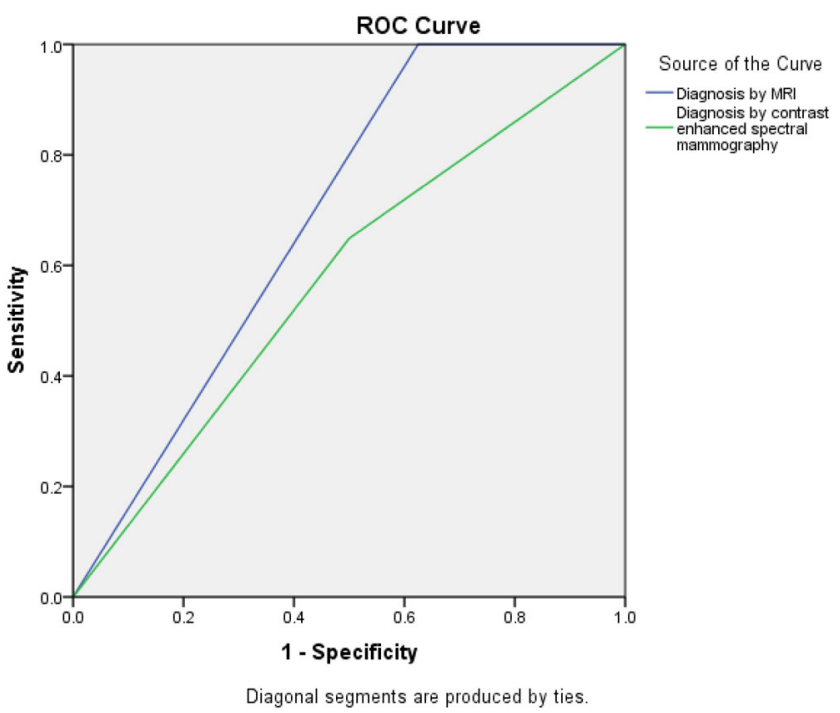

In ROC curve, the area under the curve for MRI $=0.69$ and the area under the curve for mammography $=0.57$

second group between 5 and $10 \mathrm{~mm}$ and the third group when the size is $\leq 5 \mathrm{~mm}$.

When the size of the lesion is larger than $10 \mathrm{~mm}$ (Figs. 1 and 2), the sensitivity of MRI for intraductal papilloma is still $100 \%$ but, its specificity is higher 66.7\%. In the same size group, CESM has lower sensitivity of $90.1 \%$ and specificity $0 \%$ as compared to MRI (Table 7).

The area under the ROC curve for MRI $=0.83$ and the area under the curve for $\mathrm{CESM}=0.46$.

In the second size group between 5 and $10 \mathrm{~mm}$ (Fig. 3), the sensitivity of MRI for intraductal papilloma is still $100 \%$ but, its specificity is lower $50 \%$. In the same size group, CESM has sensitivity $63.6 \%$ and specificity $50 \%$ (Table 8 ). The area under the $\mathrm{ROC}$ curve for MRI $=0.75$ and the area under the curve for $\mathrm{CESM}=0.43$.

In the third size group $\leq 5 \mathrm{~mm}$, the sensitivity of MRI for intraductal papilloma is still $100 \%$ but, its specificity is much lower $0 \%$. In the same size group, CESM has low sensitivity of $0 \%$ and high specificity $100 \%$ (Table 9). The area under the ROC curve for MRI $=0.5$ and the area under the curve for CESM = 0.5 . 


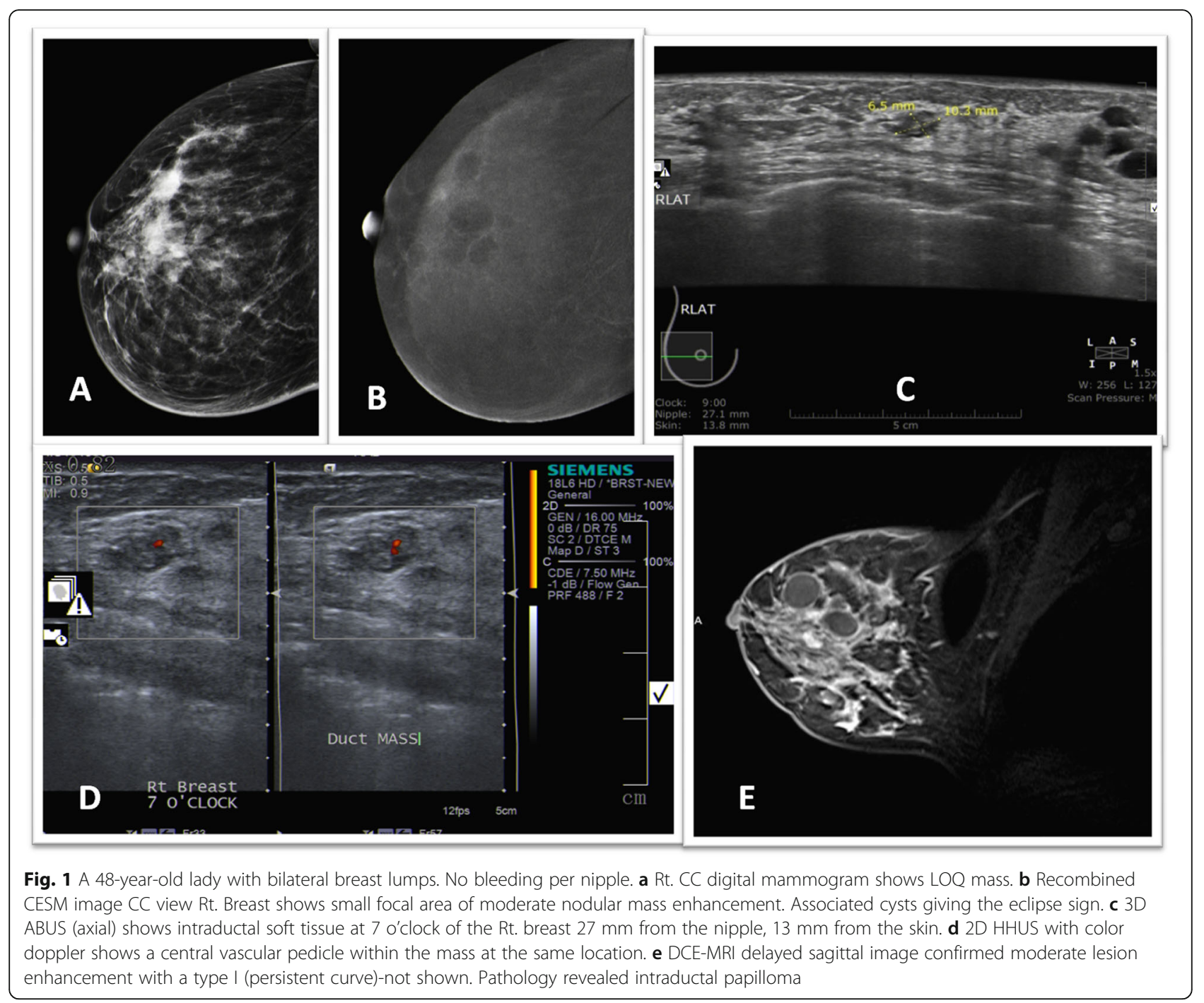

Eight cases were diagnosed as non-papillomas by histopathology (Table 2) as follows:

- Invasive duct carcinoma: this showed avid contrast enhancement on the CESM and DCE-MRI with a type III curve and irregular margin, suggesting that the lesion is malignant. It was considered as a true negative case for MRI and a false positive case for CESM (Fig. 4).

- Non-caseating granulomatous mastitis: showed multiple nodular enhancing lesions with non-mass segmental heterogenous enhancement on both CESM and DCE-MRI suggestive of multiple intraductal papillomatosis (Fig. 5).

- Intraductal papillary cystic carcinoma: this showed mild ductal enhancement on CESM and the intraductal component was not seen, while DCE-MRI showed a small $(4 \mathrm{~mm})$ intraductal enhancing lesion.
- Two cases of organized hematoma; on CESM, one of them was reported as an intraductal papilloma and the second case was not detected. On DCE-MRI, both lesions were initially hyperintense on T1WI with blooming on Gradient sequences and marginal enhancement. So, DCEMRI gave a correct diagnosis of the organized hematoma.

- Two false-positive cases on MRI due to foci of parenchymal enhancement with no positive histopathology.

- One case of fibroadenoma which was diagnosed as intraductal papilloma on CESM and DCE-MRI.

\section{Data management}

The data were collected, tabulated, and statistically analyzed using an IBM personal computer with Statistical Package of Social Science (SPSS) version 20 and 


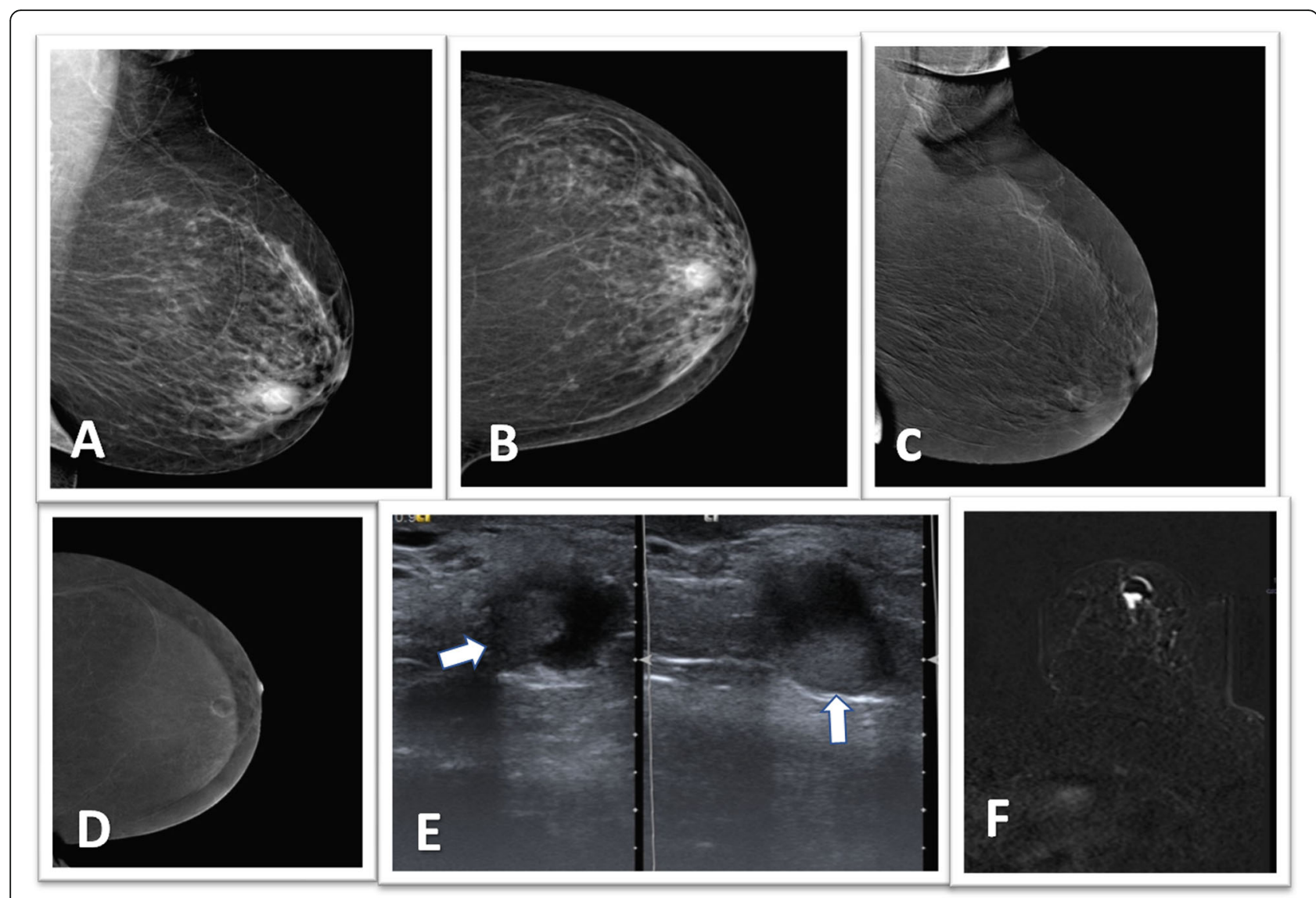

Fig. 2 A 50-year-old lady presenting with left bleeding per nipple with palpable lump. a, b MLO and CC digital mammogram shows lower welldefined nodule at 6 o'clock. c, d Recombined CESM shows well-defined rounded mass lesion with faint marginal enhancement and mildly enhancing component within. e Ultrasound revealed a complex cystic lesion with an intra-cystic soft tissue component. f DCE-MRI shows the cystic lesion with intensely enhancing soft tissue component of dynamic curve type II. Pathology revealed atypical intraductal papilloma

Epi Info 2000 programs, where the following statistics were applied.

a- Descriptive statistics: in which quantitative data were presented in the form of mean $(\bar{X})$, standard deviation (SD)

b- Range and qualitative data were presented in the form numbers and percentages (\%).

c- Analytical statistics:

- Chi-squared test $\left(\chi^{2}\right)$ was used to study the association between two qualitative variables

- Student's $t$ test is a test used for comparison between two groups having quantitative parametric variables while

- Receiver-operating characteristic (ROC) curve was used to determine cutoff points, sensitivity, and specificity for quantitative variables of interest and $2 \times 2$ tables used for calculation of PPV, NPV, and diagnostic accuracy.
- $P$ value of (>0.05) was considered not statistically significant.

- $P$ value of $(\leq 0.05)$ was considered statistically significant.

- $P$ value of $(\leq 0.001)$ was considered statistically highly significant.

\section{Discussion}

For the detection and staging of breast cancer, DCEMRI is the gold standard method, but it has many limitations as low specificity, high cost, long duration of examination time, and limited availability. CESM is considered now a relatively new imaging modality which can provide both anatomic and functional information of the breast lesion similar to DCE-MRI [12-14].

The advantage of contrast mammography over DCEMRI is that it is more affordable, easier to perform and takes much less time as compared to MRI. Also, the higher sensitivity of DCE-MRI is accused by numerous false-positive foci of enhancement [13]. 
Table 7 Diagnosis of papilloma by MRI \& CESM when the size of the lesion $>10 \mathrm{~mm}$

\begin{tabular}{|c|c|c|c|c|c|c|}
\hline & $\begin{array}{l}\text { Positive by } \\
\text { MRI }\end{array}$ & $\begin{array}{l}\text { Negative } \\
\text { by MRI }\end{array}$ & Total & $\begin{array}{l}\text { Positive by } \\
\text { CESM }\end{array}$ & $\begin{array}{l}\text { Negative } \\
\text { by CESM }\end{array}$ & Total \\
\hline Papilloma & 22 & 0 & 22 & 20 & 2 & 22 \\
\hline Non papilloma & 1 & 2 & 3 & 3 & 0 & 3 \\
\hline Total & 23 & 2 & 25 & 23 & 2 & 25 \\
\hline Sensitivity & \multicolumn{3}{|c|}{$(22 / 22) * 100=100 \%$} & \multicolumn{3}{|c|}{$(20 / 22) * 100=90.1 \%$} \\
\hline Specificity & \multicolumn{3}{|c|}{$(2 / 3) * 100=66.7 \%$} & \multicolumn{3}{|c|}{$(0 / 3) * 100=0 \%$} \\
\hline False positive & \multicolumn{3}{|c|}{$(1 / 3) * 100=33.3 \%$} & \multicolumn{3}{|c|}{$(3 / 3)^{*} 100=100 \%$} \\
\hline False negative & \multicolumn{3}{|c|}{$(0 / 22) * 100=0 \%$} & \multicolumn{3}{|c|}{$(2 / 22) * 100=10.9 \%$} \\
\hline $\begin{array}{l}\text { Positive } \\
\text { predictive value }\end{array}$ & \multicolumn{3}{|c|}{$(22 / 23) * 100=95.7 \%$} & \multicolumn{3}{|c|}{$(20 / 23) * 100=86.9 \%$} \\
\hline $\begin{array}{l}\text { Negative } \\
\text { predictive value }\end{array}$ & \multicolumn{3}{|c|}{$(2 / 2)^{*} 100=100 \%$} & \multicolumn{3}{|c|}{$(0 / 2) * 100=0 \%$} \\
\hline
\end{tabular}

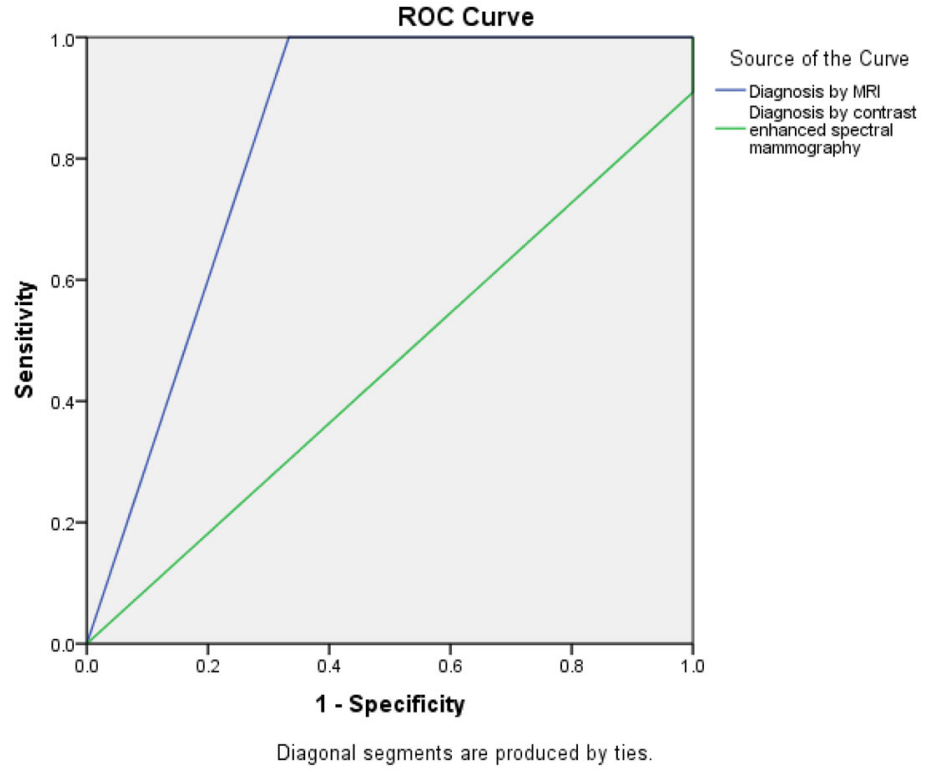

In ROC curve, the area under the curve for MRI=0.83and the area under the curve for mammography $=0.46$

CESM can be done when there are contraindications to DCE-MRI (pacemakers, claustrophobia, etc.). It is considered an affordable, efficient, and more accessible alternative to DCE-MRI. It can be easily done in a daily workflow when conventional digital mammography and ultrasound are inconclusive for suspicious findings [15].

One of the limitations of CESM is that it does not provide the possibility of enhancement kinetic analyses like DCE-MRI [15].

In our study, DCE-MRI has a higher sensitivity (100\%) for the detection of intraductal papilloma as compared to the sensitivity of CESM (64.9\%) regardless of the size of the lesion. However, DCE-MRI has a lower specificity of $37.5 \%$ as compared to the specificity of CESM (50\%).

When we sub-grouped the cases according to the size of the lesion, we found that DCE-MRI sensitivity is high in all groups (100\%), while its specificity became less when the size of the lesion was smaller and was $0 \%$ when the size of the lesion was less than 5 $\mathrm{mm}$.

On the other hand, the sensitivity of CESM was higher when the size of the lesion was more than 10 


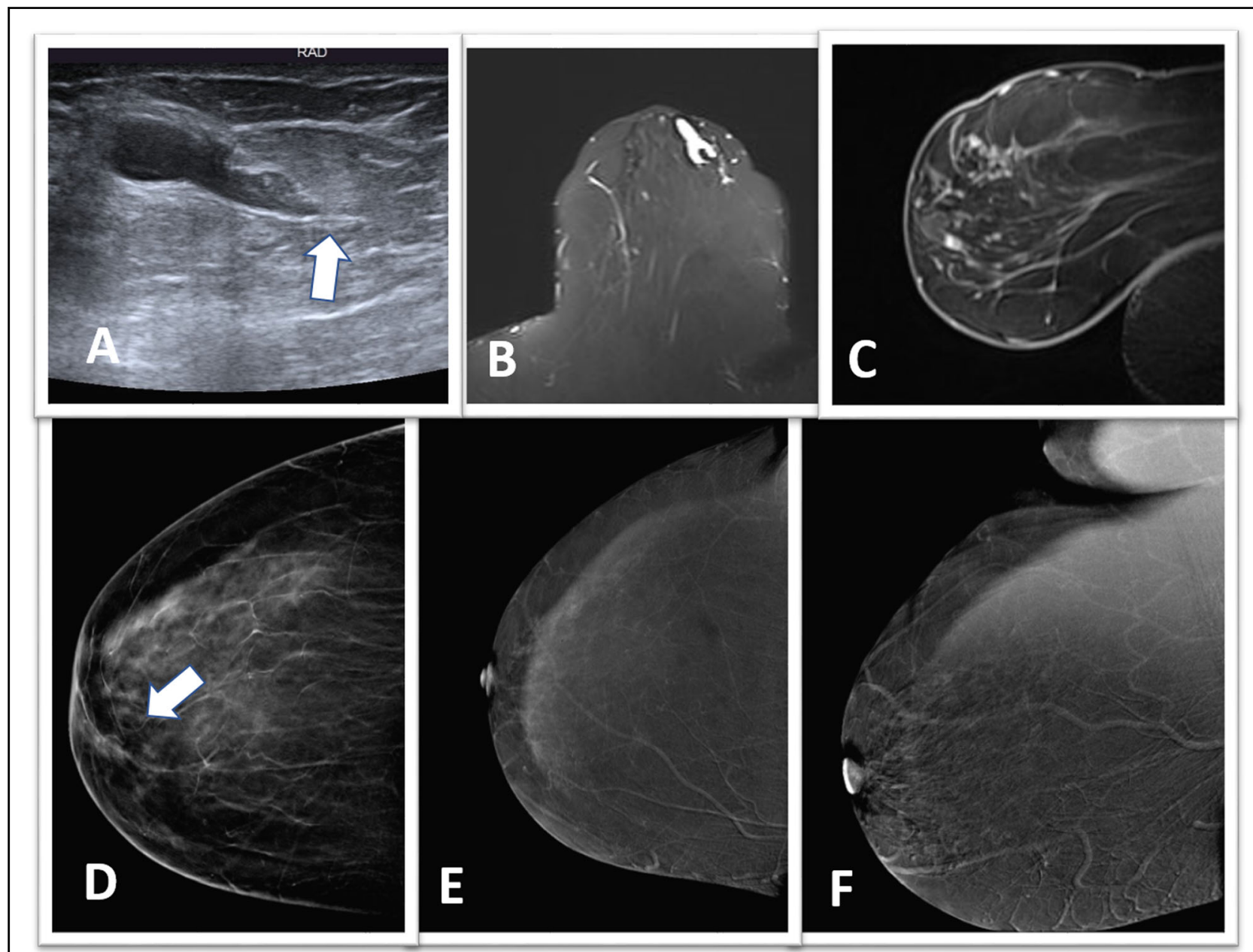

Fig. 3 A 54-year-old lady presenting with right nipple yellowish discharge. a 2D HHUS revealed focal dilated duct at 5 o'clock with an intraductal echogenic soft tissue component. b MRI-TIRM revealed dilated branching duct of high signal. c Sagittal DCE-MRI revealed an intraductalenhancing mass lesion of dynamic curve type I. d CC digital mammogram shows a linear dense structure at the lower inner quadrant (arrow). On recombined CESM (e, f), there was no enhancement. Pathology revealed: intraductal papilloma

mm (90.1\%) with low specificity (0\%). For lesions between 5 and $10 \mathrm{~mm}$, the sensitivity of CESM became less (63.6\%) and its specificity was higher (50\%).

In lesions less than $5 \mathrm{~mm}, \mathrm{CESM}$ did not detect any intraductal lesion with the sensitivity $0 \%$ and its specificity $100 \%$.

Previously published studies compared CESM and DCE-MRI in breast cancer or BIRADS 4 lesions [12, 14, $16,17]$, with no previous study done on the comparison between both CESM and DCE-MRI in intraductal papilloma.

Jochelson et al. did a prospective study and concluded that CESM has a lower sensitivity and higher specificity when compared to breast MRI [9].

Yousefa et al. agreed with Houssami et al. [18]. They concluded that CESM has lower sensitivity and higher specificity for cancer breast. The low specificity of breast MRI led to further un-necessary work-up and unindicated biopsies [14].

DCE-MRI has been useful in the diagnosis of breast tumors. Previous studies have found that enhancement intensities measured $2 \mathrm{~min}$ after contrast administration are significantly higher in malignant than benign lesions [19]. Some authors reported that the enhancement of intraductal papillomas on DCE-MRI was not obvious. The initial enhancement rate was not more than $100 \%$ and in other cases was less than $10 \%[20]$.

In our study, the enhancement of intraductal lesions was variable on both CESM and DCE-MRI. It varied from mild to intense enhancement and from marginal to homogenous enhancement. All pathologically proven papilloma cases showed a type I (28/37cases; $75 \%)$ and type II (7/37 cases; 20\%) dynamic curves 
Table 8 Diagnosis of papilloma by MRI \& CESM when the size of the lesion between 5-10mm

\begin{tabular}{|c|c|c|c|c|c|c|}
\hline & $\begin{array}{l}\text { Positive by } \\
\text { MRI }\end{array}$ & $\begin{array}{l}\text { Negative } \\
\text { by MRI }\end{array}$ & Total & $\begin{array}{l}\text { Positive by } \\
\text { CESM } \\
\end{array}$ & $\begin{array}{l}\text { Negative } \\
\text { by CESM }\end{array}$ & Total \\
\hline Papilloma & 11 & 0 & 11 & 4 & 7 & 11 \\
\hline Non papilloma & 1 & 1 & 2 & 1 & 1 & 2 \\
\hline Total & 12 & 1 & 13 & 5 & 8 & 13 \\
\hline Sensitivity & \multicolumn{3}{|c|}{$(11 / 11) * 100=100 \%$} & \multicolumn{3}{|c|}{$(7 / 11) * 100=63.6 \%$} \\
\hline Specificity & \multicolumn{3}{|c|}{$(1 / 2) * 100=50 \%$} & \multicolumn{3}{|c|}{$(1 / 2) * 100=50 \%$} \\
\hline False positive & \multicolumn{3}{|c|}{$(1 / 5) * 100=50 \%$} & \multicolumn{3}{|c|}{$(1 / 2) * 100=50 \%$} \\
\hline False negative & \multicolumn{3}{|c|}{$(0 / 11)^{*} 100=0 \%$} & \multicolumn{3}{|c|}{$(4 / 11) * 100=36.4 \%$} \\
\hline $\begin{array}{l}\text { Positive } \\
\text { predictive value }\end{array}$ & \multicolumn{3}{|c|}{$(11 / 12)^{*} 100=91.7 \%$} & \multicolumn{3}{|c|}{$(4 / 5) * 100=80 \%$} \\
\hline $\begin{array}{l}\text { Negative } \\
\text { predictive value }\end{array}$ & \multicolumn{3}{|c|}{$(1 / 1)^{*} 100=100 \%$} & \multicolumn{3}{|c|}{$(1 / 8)^{*} 100=12.5 \%$} \\
\hline
\end{tabular}

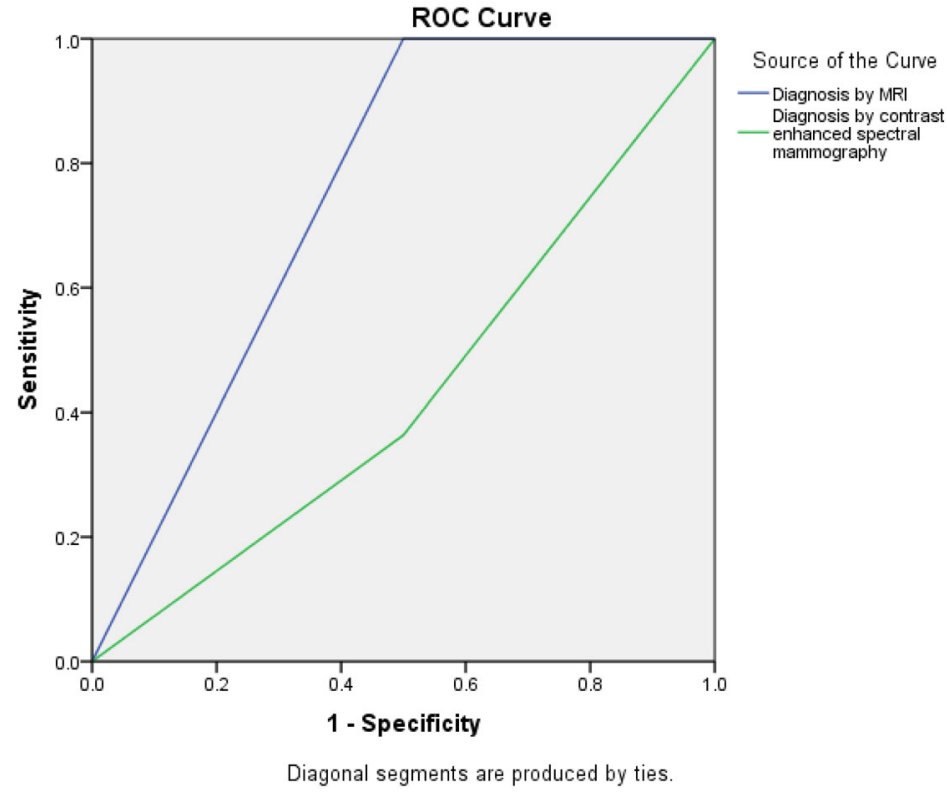

The area under the curve for $\mathrm{MRI}=0.75$ and the area under the curve for mammography $=0.43$

while lesions giving a type III curve (2/37 cases; $5 \%)$; proved to be associated with another underlying pathology; granulomatous mastitis in one case and inflammatory changes in another.

Zhu et al. [1] noted that rapid initial enhancement was seen in both intraductal papillomas and invasive carcinomas, but the early-phase enhancement rate of intraductal papilloma was significantly lower than that of invasive carcinomas $(p<0.05)$. The fibrovascular bundle structure of intraductal papilloma might provide the histologic explanation for the uptake of contrast material [1]. The time-intensity curve is an important criterion to distinguish between patterns of enhancement of benign and malignant breast lesions $[19,21]$. Washout curves are an important predictor of malignancy, whereas persistent and plateau patterns suggest benign lesions [21].

The reported MR enhancement patterns of intraductal papillomas are variable. Daniel et al. [22] studied the DCE-MRI performance of intraductal papillomas and reported that most enhancing papillomas showed a plateau curve. Krämer et al. [20] concluded that benign papillomas did not show patterns of enhancement suggestive of malignancy. In their study, all papillomas 
Table 9 Diagnosis of papilloma by MRI \& CESM when the size of the lesion $\leq 5 \mathrm{~mm}$

\begin{tabular}{|c|c|c|c|c|c|c|}
\hline & $\begin{array}{l}\text { Positive by } \\
\text { MRI }\end{array}$ & \begin{tabular}{|l|} 
Negative \\
by MRI
\end{tabular} & Total & $\begin{array}{l}\text { Positive by } \\
\text { CESM }\end{array}$ & $\begin{array}{l}\text { Negative } \\
\text { by CESM }\end{array}$ & Total \\
\hline Papilloma & 4 & 0 & 4 & 0 & 4 & 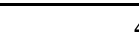 \\
\hline Non papilloma & 3 & 0 & 3 & 0 & 3 & . \\
\hline Total & 7 & 0 & 7 & 0 & 7 & ? \\
\hline Sensitivity & \multicolumn{3}{|c|}{$(4 / 4) * 100=100 \%$} & \multicolumn{3}{|c|}{$(0 / 4) * 100=0 \%$} \\
\hline Specificity & \multicolumn{3}{|c|}{$(0 / 3)^{*} 100=0 \%$} & \multicolumn{3}{|c|}{$(3 / 3)^{*} 100=100 \%$} \\
\hline False positive & \multicolumn{3}{|c|}{$(3 / 3)^{*} 100=100 \%$} & \multicolumn{3}{|c|}{$(0 / 3)^{*} 100=100 \%$} \\
\hline False negative & \multicolumn{3}{|c|}{$(0 / 4)^{*} 100=0 \%$} & \multicolumn{3}{|c|}{$(4 / 4) * 100=100 \%$} \\
\hline \begin{tabular}{|l|} 
Positive \\
predictive value
\end{tabular} & \multicolumn{3}{|c|}{$(4 / 7) * 100=57.1 \%$} & \multicolumn{3}{|c|}{$(0 / 0)^{*} 100=0 \%$} \\
\hline $\begin{array}{l}\text { Negative } \\
\text { predictive value }\end{array}$ & \multicolumn{3}{|c|}{$(0 / 0)^{*} 100=0 \%$} & \multicolumn{3}{|c|}{$(3 / 7)^{*} 100=42.9 \%$} \\
\hline
\end{tabular}

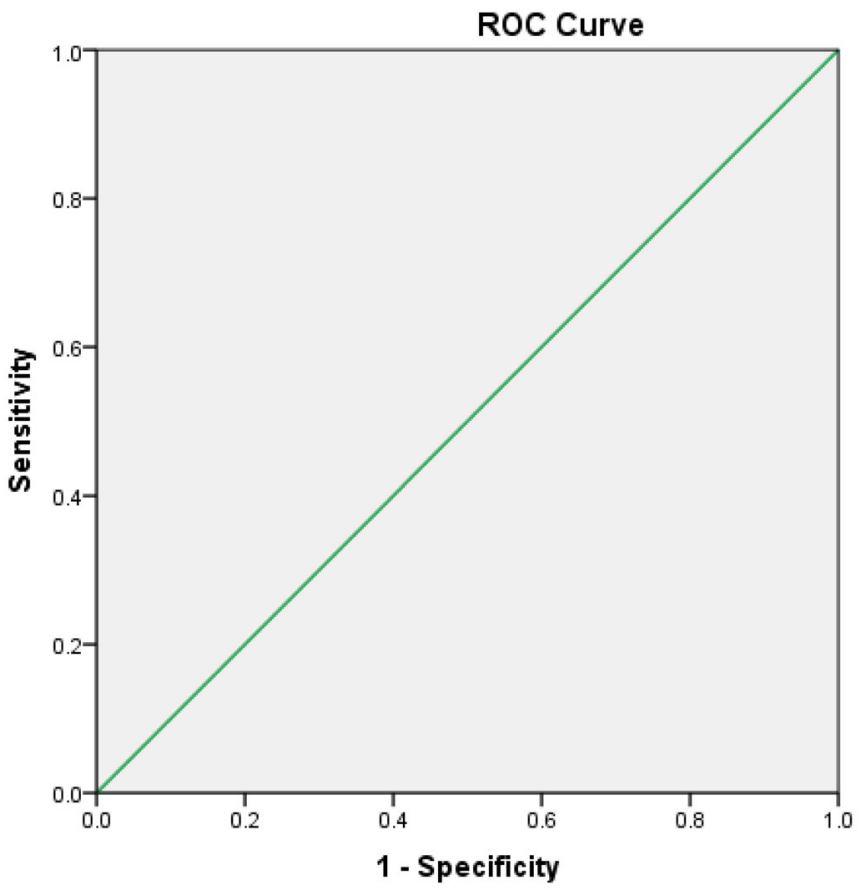

Diagonal segments are produced by ties.

The area under the curve for $\mathrm{MRI}=0.5$ and the area under the curve for mammography $=0.5$

showed rapid initial enhancement. Additionally, $72 \%$ of intraductal papillomas and $69 \%$ of invasive cancers revealed washout curves, with no significant difference between the two groups [20]. However, in another study, the dynamic curve of papillomas had a higher percentage of type III (washout) and no type I (progressive) delayed kinetic patterns of enhancement [1].

Zhu et al. showed a significant difference in the evolution of enhancement patterns in the periphery and center of intraductal papillomas versus carcinomas. They found that on DCE-MRI, most intraductal papillomas showed homogeneous or heterogeneous enhancement in the early phase and changed to rim enhancement in the delayed phase. This was scarcely seen in invasive carcinomas. This is suggested as a helpful sign in the diagnosis of intraductal papilloma of the breast [1].

Graziani et al. found that there is a variable morphologic appearance of benign papillomas on DCE-MRI as it can 


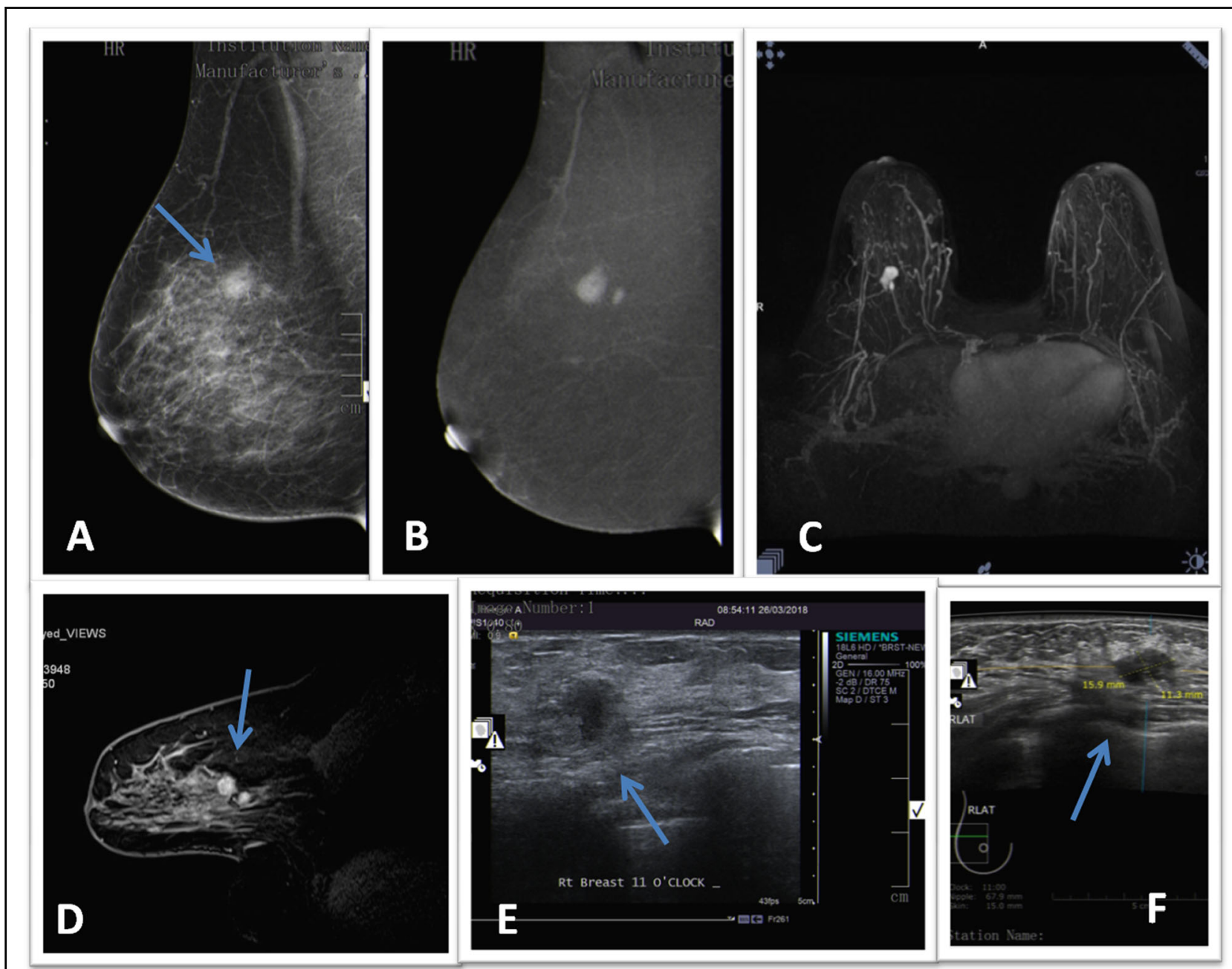

Fig. 4 A 44-year-old lady presenting with an enlarging right breast lump. a Rt. MLO digital mammogram shows UOQ nodule within glandular tissue. b Recombined CESM clearly shows two homogenously enhancing well defined nodules (avid enhancement) within a non-enhancing background. c DCE-MRI-MIP image shows the enhancing lesion within the breast with an adjacent vessel. $\mathbf{d}$ Delayed DCE sagittal MRI shows the two enhancing nodules within a glandular breast parenchyma. e, f 2D HHUS and 3D ABUS respectively shows a single complex cystic lesion. Pathology revealed: invasive ductal carcinoma grade I

be occult lesion, "fibroadenoma-like" lesion or even "tumor-like" lesions. Also, they detected different MRI enhancement patterns, from mild, slow enhancement to early, strong, early enhancement with a plateau or a washout enhancement pattern. The kinetic curve of strongly enhancing lesion usually types 2 or 3 curves that make it very similar to malignant lesions [23].

In our study, since we concentrated on papilloma cases (37/45 cases; 82\%), we found this pattern of marginal enhancement in (27/37 cases; $73 \%)$ of MRI detected papillomas while others $(10 / 37$ cases; $7 \%)$ showed homogenous enhancement with no ring pattern in the delayed phase. This can be attributed to the small size of an intraductal papilloma which makes it more difficult to show this pattern.

Lobbes et al. compared CESM and MRI in the assessment of the size of the breast tumor. They found that
CESM is good for measuring the size of the tumor while MRI did not add to the assessment of tumor size [24].

In a study done by Rotha et al., the smallest enhancing lesion detected on post-contract images was a $4 \mathrm{~mm}$ DCIS. This lesion was identified on both CESM and BMRI [13].

While in another study, breast MRI and CESM were found similar in the assessment of local extent of disease, lesion size, and multifocal/multicentric involvement, but they found CESM not the optimal study for some benign lesions as intraductal papillomas, small fibroadenomas, and radial scars [25].

In our study, the mean size of lesions was $11.1 \pm 4.6$ $\mathrm{mm}$ on DCE-MRI and $13.8 \pm 3.4 \mathrm{~mm}$ on CESM with statistical significance $P=0.005^{*}(\leq 0.05)$ (Table 4). The smallest detected size on DCE-MRI was $4 \mathrm{~mm}$.

Regarding using mass and non-mass enhancement as morphology descriptors in CESM as described by Kamal 

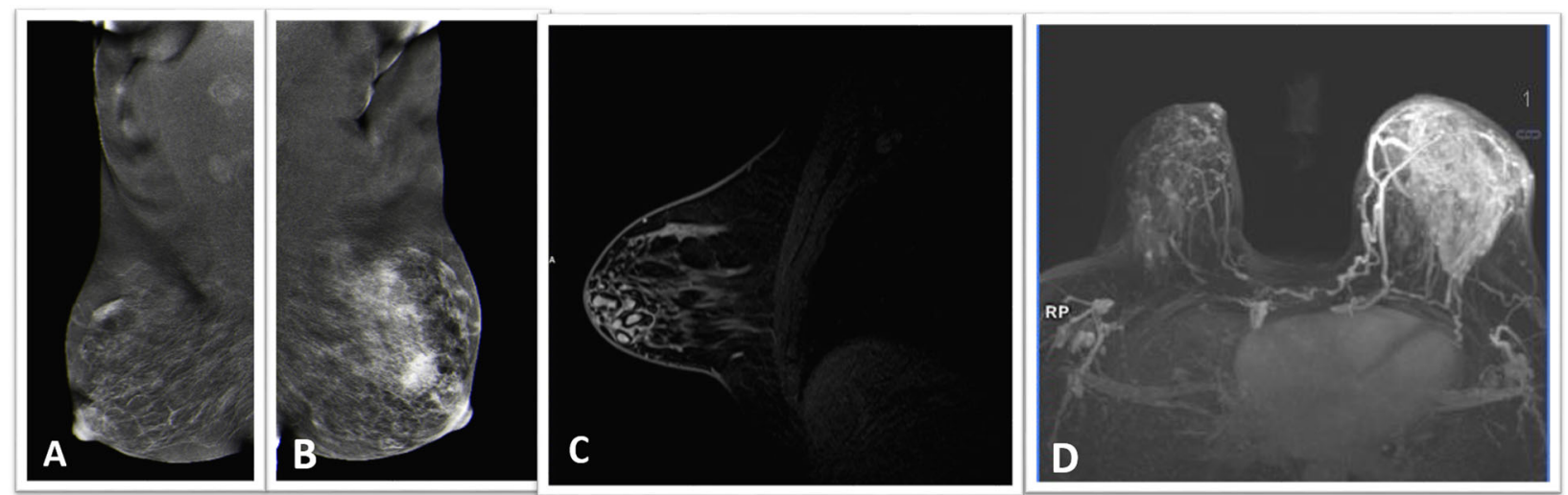

Fig. 5 A 54-year-old lady presenting with bilateral breast discharge. a, b Rt. and Lt. MLO recombined CESM; right breast shows an UOQ area of intense non-mass tubular enhancement with tubular distribution. Left shows an area of segmental enhancement. c Sagittal delayed DCE-MRI of the right breast shows multiple intraductal-enhancing retroareolar masses not seen on CESM. $\mathbf{d}$ CE-MRI-MIP image shows the area of segmental non-mass enhancement within the left breast. Histopathology revealed bilateral papillomatosis with granulomatous mastitis

et al. in 2015 [26], we found this difficult in case of small lesions as intraductal papillomas especially those less than $15 \mathrm{~mm}$ in size. In our study, only 4 cases $(4 / 45$ cases) $9 \%$ showed a well-defined mass enhancement on CESM even when MRI showed a well-defined enhancing nodule; of these, two were benign intraductal papillomas of size $15 \mathrm{~mm}$, one was invasive ductal carcinoma, and the fourth was papillary cystic carcinoma. In our study, of all the 37 papillomas diagnosed on histopathology, only 20 showed contrast enhancement (54\%) on CESM of which 18 cases (49\%) showed non-mass enhancement either focal or ductal distribution. This may be attributed to the small size of the lesions; however, all papilloma cases showing regional or segmental enhancement on CESM proved to be associated with an underlying pathology; granulomatous mastitis in one case and inflammatory changes in another.

\section{Limitations of this study}

Our study has some limitations; small sample size may affect results. More researches with larger number of cases may be of value. All patients enrolled in the study were initially diagnosed as papillomas on MG and ultrasound, which may have incurred some entry bias in favor of papilloma compared to other breast pathology. Diffusion was not included as a point of comparison in MRI criteria which might prove to be of value in further research.

\section{Conclusion}

We conclude that MRI is still superior to CESM for the diagnosis of intraductal lesions whatever the size of the lesion, especially when the size is less than $5 \mathrm{~mm}$. There is a lack of published research on CESM in diagnosing intraductal papilloma when compared with DCE-MRI.
More research on a larger number of patients is needed for a more accurate assessment of the specificity of CESM in these cases.

\section{Abbreviations}

2DHHUS: Two-dimensional hand-held ultrasound; BI-RADS: Breast ImagingReporting and Data System; CC: Craniocaudal; CE-MRI: Contrast-enhanced magnetic resonance imaging; CESM: Contrast-enhanced spectral mammography; DCIS: Ductal carcinoma in situ; DWI: Diffusion-weighted imaging; FFDM: Full-field digital mammography; FNAC: Fine needle aspiration cytology; FSE: Fast spin-echo; IDC: Invasive ductal carcinoma; MG: Mammography; MIP: Maximum intensity projections; MLO: Mediolateral oblique; MPR: Multi-planar reconstruction; TIRM: Turbo inversion recovery magnitude

\section{Acknowledgements}

Thanks are due to patients, radiographers, and histopathologists who contributed in this work.

\section{Authors' contributions}

Equal sharing in data collection and analysis, manuscript writing and editing by the three authors. RA and RY did the initial manuscript writing and data analysis. LA shared in writing, editing and revising data and manuscript. All authors have read and approved the final manuscript.

\section{Funding}

Self-funding.

Availability of data and materials

All data and materials used in this research are available.

Ethics approval and consent to participate

The study protocol was approved by the Local Ethics Committee. Vancouver ethical standards were followed in the conduct of the study. No available ethics' committee reference number. All patients signed written consent to perform the procedure.

\section{Consent for publication}

All patients included in this research gave written informed consent to publish the data contained and/or analyzed within this study.

Competing interests

The authors declare they have no competing interests. 


\section{Author details}

${ }^{1}$ Radiology Department, Faculty of Medicine, Cairo University, Cairo, Egypt.

${ }^{2}$ Diagnostic Radiology, Faculty of Medicine, Menofia University, Menofia Egypt.

Received: 2 October 2019 Accepted: 19 December 2019

Published online: 04 February 2020

\section{References}

1. Zhu Y, Zhang S, Liu P et al (2012) Solitary intraductal papillomas Of the breast: MRI features and differentiation from small invasive ductal carcinomas. AJR 199:936-942

2. Dhull V, Sen J, Yadav R, et al.( 2016) Case series: imaging features of intraductal papillomas in patients presenting as nipple discharge. Int J Res Med Sci. Jul; 4 (7): 2878-2882).

3. Eiada R, Chong J, Kulkarni S et al (2012) Papillary lesions of the breast: MRI, ultrasound, and mammographic appearances. AJR 198:264-271

4. Brookes MJ, Bourke AG (2008) Radiological appearances of papillary breast lesions. Clin Radiol 63:1265-1273

5. Francis A, England D, Rowlands D, Bradley S (2002) Breast papilloma: mammogram, ultrasound and MRI appearances. Breast 11:394-397

6. Lam WW, Chu WC, Tang AP, Tse G, Ma TK (2006) Role of radiologic features in the management of papillary lesions of the breast. AJR 186:1322-1327

7. Aljarrah A, Malik KA, Jamil H et al (2015) Diagnostic dilemmas in Intraductal papillomas of the breast - Experience at Sultan Qaboos University Hospital in the Sultanate of Oman. Pak J Med Sci 31:2

8. Friedewald SM, Rafferty EA, Rose SL et al (2014) Breast cancer screening using tomosynthesis in combination with digital mammography. JAMA 311(24):2499-2507

9. Jochelson MS, Dershaw DD, Sung JS et al (2013) Bilateral contrast-enhanced dual-energy digital mammography: feasibility and comparison with conventional digital mammography and MR imaging in women with known breast carcinoma. Radiology 266(3):743-751

10. Francescone MA, Jochelson MS, Dershaw DD et al (2014) Low energy mammogram obtained in contrast-enhanced digital mammography (CEDM) is comparable to routine full-field digital mammography (FFDM). Eur J Radiol 83(8):1350-1355

11. Dromain C, Balleyguier C, Adlera G, Garbay JR, Delaloge S (2009) Contrastenhanced digital mammography. Eur Radiol 69:34-42

12. Nekhlyudov L, Kiarsis K, Elmore JG (2009) MRI of the breast: does the internet accurately report its beneficial uses and limitations? Breast J 15:189-193

13. Rotha L.R, Germaineb P, Renc S, et al. (2017) Contrast-enhanced spectral mammography (CESM) versus breast magnetic resonance imaging (MRI): A retrospective comparison in 66 breast lesions. Diagn Interv Imaging. Feb; 98(2):113-123. https://doi.org/10.1016/j.diii.2016.08.013

14. Yousef AF, Khater HM, Jameel LM (2018) Contrast-enhanced spectral mammography versus magnetic resonance imaging in the assessment of breast masses. Benha Med J 35:5-12

15. Roganovic D, Djilas D, Vujnovic S et al (2015) Breast MRI, digital mammography and breast tomosynthesis: comparison of three methods for early detection of breast cancer. BosnJ Basic Med Sci 15:64-68

16. Froeling V, Diekmann F, Renz DM, Fallenberg EM, Steffen IG, Diekmann S et al (2013) Correlation of contrast agent kinetics between iodinated contrast-enhanced spectral tomosynthesis and gadolinium-enhanced MRI of breast lesions. Eur Radiol 23:1528-1536

17. Xing D, Lv Y, Sun B et al (2019) Diagnostic value of contrast-enhanced spectral mammography in comparison to magnetic resonance imaging in breast lesions. J Comput Assist Tomogr 43:245-251

18. Houssami N, Ciatto S, Macaskill P et al (2008) Accuracy and surgical impact of magnetic resonance imaging in breast cancer staging: systematic review and meta-analysis in detection of multifocal and multicentric cancer. J Clin Oncol 26:3248-3258

19. Kuhl CK, Mielacareck $P$, Klaschik S et al (1999) Dynamic breast MR imaging: are signal intensity time course data useful for differential diagnosis of enhancing lesions? Radiology 211:101-110

20. Krämer SC, Rieber A, Görich J et al (2000) Diagnosis of papillomas of the breast: value of magnetic resonance mammography in comparison with galactography. Eur Radiol 10:1733-1736

21. Ikeda DM, Hylton NM, Kuhl CK, et al. (2003) BI-RADS: magnetic resonance imaging. In: American College of Radiology. Breast imaging reporting and data system: (BI-RADS), 4th Ed. Reston, VA: American College of Radiology;:17-95.
22. Daniel BL, Gardner RW, Birdwell RL et al (2003) Magnetic resonance imaging of intraductal papilloma of the breast. Magn Reson Imaging 21:887-892

23. Graziani G, Linda A, Gualano A et al (2012) Magnetic resonance imaging appearance of benign papillomas of the breast. https://doi.org/10.1594/ECR/ C-0773

24. Lobbes MB, Lalji UC, Nelemans PJ et al (2015) The Quality of tumor size assessment by contrast-enhanced spectral mammography and the benefit of additional breast MRI. J Cancer 6:144

25. Mokhtar O, Mahmoud S (2014) Can contrast enhanced mammography solve the problem of dense breast lesions? Egyptian J Radiol Nuclear Med 45:1043-1052

26. Kamal R, Helal M,Wessam R, et al. (2015) Contrast-enhanced spectral mammography: Impact of the qualitative morphology descriptors on the diagnosis of breast lesions. Eur J Radiol, Vol. 84, 1049-1055 DOI: https://doi. org/https://doi.org/10.1016/j.ejrad.2015.03.005

\section{Publisher's Note}

Springer Nature remains neutral with regard to jurisdictional claims in published maps and institutional affiliations.

\section{Submit your manuscript to a SpringerOpen ${ }^{\circ}$ journal and benefit from:}

- Convenient online submission

- Rigorous peer review

- Open access: articles freely available online

- High visibility within the field

- Retaining the copyright to your article

Submit your next manuscript at $>$ springeropen.com 